Volume 8. No. 5, May 2020

International Journal of Emerging Trends in Engineering Research

Available Online at http://www.warse.org/IJETER/static/pdf/file/ijeter21852020.pdf

https://doi.org/10.30534/ijeter/2020/21852020

\title{
Information Flows in Hydraulic Structures
}

\author{
Safar Z. Zainudinov ${ }^{1}$, Maksim S. Paliivets ${ }^{2}$, Bakhtier S. Zainudinov ${ }^{3}$
}

: of Technical Sciences, Associate Professor of the Department of Information Technologies in Agriculture, Russian State Agrarian University - Moscow Timiryazev Agricultural Academy, Moscow, Russia; pol123987@mail.ru ${ }^{2}$ Candidate of Technical Sciences, Associate Professor of the Department of Information Technologies in Agriculture, Russian State Agrarian University - Moscow Timiryazev Agricultural Academy, Moscow, Russia; maxim6663@mail.ru ${ }^{3}$ Civil Engineer, LLC “RUSFEN”, Gubkinsky, Yamalo-Nenets Autonomous Okrug, Russia; zaynudinovbakhtiyor@gmail.com

\begin{abstract}
The article discusses the theoretical aspects and practical implementation of the definition, identification and assessment of information flows and the content of information in the objects of hydraulic structures. The processes of the occurrence of possible additional internal volume surfaces and the transfer of information between them are described on the basis of general physical theories of the interaction of condensed matter, using a sequential analysis of the state of the emerging rubbing surfaces due to their dynamic interaction. For this purpose, objects of hydraulic structures, regardless of their classifications, were divided into naturally formed and artificially formed surfaces. The degrees of interaction between them were determined on the basis of a probabilistic approach to the energy states of surfaces. In this case, the fact that the surfaces have mainly two types of energy levels was taken into account. Energy levels are loosely coupled molecular energy levels and pronounced magnetic and electrical energy levels of electrons, atoms, molecules. The first relates to hard artificial surfaces, and the second to natural surfaces. In addition, the properties of the surfaces were associated with the homogeneity of the materials of the objects, which affects the activity of the emerging boundary layers. The proposed methodology determines the greatest probability of destruction resulting from the dynamic interaction of the surfaces of hydraulic objects using information technology. The article provides some examples of the use of modern information systems for organizing various work models based on the proposed methodology for some objects of hydraulic structures. The reliability of the achieved results of the application of the technique is supported by well-known mathematical laws.
\end{abstract}

Key words: hydraulic structures, an object, naturally formed objects, artificially formed objects, contacting surfaces, volumetric processes, surface.

\section{INTRODUCTION}

Despite the fact that hydraulic structures have scattered and various characteristics according to classification, class, functional purpose, location, which are very different from each other, hydraulic structures can be divided into naturally formed and artificially formed objects in order to effectively manage them. Separation of objects in this way makes it possible to use a flexible approach when selecting a tool for studying the composition of information flows and compiling on their basis the most important system of information flows for the life of a hydraulic structure. Some attempts in this area were made when studying the interaction of a cold surface with the surfaces of objects of a hydraulic structure [2] and their influence on the injection ability of interacting objects [7], which do not allow their results to be generalized to all objects of a hydraulic structure.

Any objects, parts and processes that are obtained by acting on them by artificial means, or by direct human actions using artificial means can be attributed to an artificially formed structure. Examples are waterfront structures, locks, dams, irrigation systems, culverts on reclamation canals [6], lintels, canals, storm sewers, and other objects, as well as objects that ensure the life of the hydraulic structure. Each of the listed objects have parameters that differ from each other. The parameters of these objects are constant, unchanged, weakly exposed to danger and parameters that are weakly destructive from the effects of the nature and duration of the life cycle. Examples of such parameters for artificially formed objects are height, length, width, volume, physicochemical properties of the material of the object.

Naturally formed objects should include all objects that are formed: 1) between the contacting surfaces of any artificially formed objects, 2) between the touching surfaces of any artificially formed and naturally formed objects, 3) between the touching surfaces of any naturally formed objects. Since these objects are in dynamic interaction with each other, the processes between them will be variable in the following characteristics: in content, in intensity, in space coverage, in the formation of a new border surface, etc.

\section{RESEARCH METHODS}

The methods of scientific analysis of information flows were used in the article, which determine the sources and receivers of information, their location, the occurrence of processes between them, the direction of information flow in the flows between sources and receivers of information. It is assumed that the use of general physical laws and 
mathematical expressions to describe processes between separated surfaces expands the capabilities of this technique in identifying processes in artificially formed objects and in naturally formed objects.

It should be noted that there is a common and distinctive between processes in artificially formed objects and in naturally formed objects. 1) The general fact is that volumetric processes occurring inside naturally formed and artificially formed objects are natural processes and are not influenced by the human factor. For example, natural processes that are not dependent on humans occur inside the walls of water bearing, water conveyance, regulatory and sluice-way structures, as well as inside any liquids that are even changed in composition (water, oils, acids, alkalis, etc.). This means that the destructive properties of internal processes are of little significance for a hydraulic structure. Therefore, when organizing a hydraulic engineering structure, one must take into account that volumetric processes are invulnerable places and their study is of little effect in terms of the impact on the life of a hydraulic engineering structure. 2) The distinguishing feature is that the processes occurring between the surfaces of all kinds of objects are artificially formed processes, since they are created artificially and give in to human influences. It is possible to control the rate of destruction of surfaces and achieve the most effective extension of the lifetime of a hydraulic structure with the same volumetric processes in objects, simulating the processes of inter-surface interaction. In other words, the processes occurring between contacting surfaces are protective layers for internal natural volumetric processes. The question arises: how can one influence inter-surface interactions? To answer this question, it is necessary to determine the sources and receivers of these processes. Obviously, the processes occur in the following directions from source to receiver, which are indicated in the table 1.

Table 1: The pattern of occurrence of information flows

\begin{tabular}{|c|c|c|}
\hline No & Source & Receiver \\
\hline 1 & $\begin{array}{c}\text { Naturally formed } \\
\text { surface }\end{array}$ & $\begin{array}{c}\text { Naturally formed } \\
\text { surface }\end{array}$ \\
\hline 2 & $\begin{array}{c}\text { Naturally formed } \\
\text { surface }\end{array}$ & $\begin{array}{c}\text { Artificially formed } \\
\text { surface }\end{array}$ \\
\hline 3 & $\begin{array}{c}\text { Artificially formed } \\
\text { surface }\end{array}$ & $\begin{array}{c}\text { Naturally formed } \\
\text { surface }\end{array}$ \\
\hline 4 & $\begin{array}{c}\text { Artificially formed } \\
\text { surface }\end{array}$ & $\begin{array}{c}\text { Artificially formed } \\
\text { surface }\end{array}$ \\
\hline
\end{tabular}

In most cases, the surfaces of artificial objects are in some way altered surfaces of solids with a certain energy of intermolecular bonds. This means that the surfaces of artificial objects are less likely to change their surface when receiving energy from a source (object). Natural objects have a larger, both quantitative and qualitative, set of energy states, therefore, and have a higher ability to change the surface of an artificial object than artificial objects to change the surface of a natural object. This probability pattern is shown in table 1: the probability decreases from top to bottom. Thus, in terms of the rate of surface destruction, the first place is occupied by interactions of the type "Naturally formed surface Naturally formed surface".
Of great importance is the energetic constituent parts of both naturally formed and artificially formed surfaces. The analysis of information in this direction shows that surfaces mainly have the following energy levels: a) weakly bound molecular energy levels; b) pronounced magnetic and electrical energy levels of electrons, atoms, molecules. The first relates to hard artificial surfaces, and the second to natural surfaces. For example, these differences can be observed by the degree of oxidizing properties of objects: oxidative properties are less pronounced on the surfaces of artificial objects than on the surface of a natural object. This means that the process of transmitting information about the state of the source to the receiver and vice versa occurs when the surfaces interact; for example, from the surface of the water to the surface of concrete, and vice versa.

Movable constituent parts that have interacting surfaces are defined. 1) Solids are homogeneous due to the following factors: a) are made of the same material; b) made of various foreign materials, but mainly of their mechanical mixtures, which are electrically neutral compared to homogeneous particles of the basic material of a solid body. 2) Liquid bodies, mainly water, are homogeneous due to the following factors: a) consist of intrinsic molecules, b) consist of mechanical, electrically neutral (compared to a liquid) foreign molecules. Based on this, it can be assumed that the surfaces of solids are composed of molecules with less mobile and free atoms and electrons than liquids. Therefore, the surfaces of solids are less active for the formation of a boundary layer than the surface of a liquid.

\section{RESULTS AND DISCUSSION}

After listing the parameters that are necessary to compile a model for ensuring the life of a hydraulic structure $[3,7$, 8 ], we see that a huge amount of data will be required for coordinated actions at all levels of management. It is also important to correctly determine the information flows between the sources and receivers indicated in table 1, with the content of information on the information parameters of messages and signals. The determination of the content of information flows, the relationship between them and the accurate compilation of information flow systems in a hydraulic structure is the guarantor of a correctly composed model close to real phenomena.

It should be noted that the collection, storage, processing and transmission of information to solve the task is impossible without the following modern information technologies: Architecture of Integrated Information Systems such as ARIS, CD-adapco STAR-CD (Computational Dynamics-Analysis \& Design Application Company Ltd), application package Solver Microsoft Excel (Microsoft Office Excel), Statistica - software package for statistical analysis, developed by StatSoft and many others.

When using the CD-adapco STAR-CD software package, which contains built-in options for setting boundary conditions covering most of the practical tasks of modeling culverts for hydraulic structures according to the main parameters, it is possible to select PROSTAR options that provide a level of spatial resolution for setting 
Safar Z. Zainudinov et al., International Journal of Emerging Trends in Engineering Research, 8(5), May 2020,1605 - 1609

boundary conditions ranging from an individual cell to the entire surface of the computational domain when designing hybrid models of culverts of hydraulic structures.

When modeling the phenomena of culverts of hydraulic structures and their resistance nodes with specified dimensions in three-dimensional geometry, the entire pressure flow of the mathematical model in CD-adapco STAR-CD is divided into a computational grid. The purpose of constructing a grid is to describe the boundary surfaces of the computational domains and to divide these volumes into subregions or cells used for the numerical solution of the differential equations of the mathematical model. In the mathematical model, the flow grid can consist of 50,000 elements, namely, cut-off polyhedra, the density of water $p=1000 \mathrm{~kg} / \mathrm{m}^{3}$, the coefficient of kinematic viscosity $v=0,0101 \cdot 10^{-4} \mathrm{~m}^{2} / \mathrm{s}$, dynamic viscosity $\mu=10,1 \cdot 10^{-4} \mathrm{~Pa} \cdot \mathrm{s}$, the given flow rate on the model $Q=21$ $l / s$ for all designs of resistance nodes $\left(\operatorname{Re}=2 \cdot 10^{5}\right)$ at temperature $t=293 \mathrm{~K}=20^{\circ} \mathrm{C}$ are taken. The flow at the entrance to the culvert should have a kinetic energy coefficient $\alpha$ close to unity.

The boundary conditions for the mathematical model were set by piezometric pressures along the entire length of the culvert structure of the hydraulic structure, which were obtained experimentally with different schemes of nodal resistances, which made it possible to set the pressure distribution.

The boundary condition at the wall when using the software product CD-adapco STAR-CD was to establish the speed $\tilde{v}_{t}$ equal to zero. However, in the models for high Reynolds numbers (the number $\operatorname{Re}=(1 \div 3) \cdot 10^{5}$ in experiments), special functions were used that are called "wall functions" to calculate changes in velocity, temperature, and turbulent energy in the wall layer. Such a practice is inevitable, since such models are incorrect in the near-wall regions, where the molecular and turbulence effects are comparable. This is also advisable, since it helps to avoid the use of a fine mesh inside the wall layer, with a corresponding increase in the system requirements of a personal computer. The representation of turbulent properties near the boundary layer through "wall functions" is a certain approximation, the accuracy of which is determined by the adequacy of the entire set of assumptions and approximations inherent in these functions to describe the real picture of the phenomenon. The main assumption on which the standard wall functions used are based are as follows:

- $\quad$ the change in speed is predominantly normal with respect to the wall and tend to one-dimensional;

- $\quad$ the effects of pressure gradients and bulk forces are negligible, tend to a uniform distribution of shear stresses in the wall layer;

shear stresses and velocity vectors merge into a single whole and are codirectional throughout the layer;

- there is a balance between the produced and diffused energy of turbulence;

- the change in the length of the turbulence scale occurs linearly.

\section{RESEARCH RESULTS}

The resulting formulas describing the cross-flow profiles as a function of the normal distance from the wall $y$ are as follows:

Speed:

$u^{+}= \begin{cases}y^{+} & , y^{+} \leq y_{m}^{+} \\ \frac{1}{\chi} \ln \left(E y^{+}\right) & , y^{+}>y_{m}^{+}\end{cases}$

where $u^{+}=\left(u-u_{W}\right) / u_{\tau},(5) ; u$ is tangential component of the flow velocity; $u_{W}$ is tangential velocity near the wall; $u_{\tau}=\left(\tau_{W} / \rho\right)^{0,5} ; \tau_{W}$ is shear stress near the wall; $y^{+}=\rho u_{\tau} y / \mu \approx \rho C_{\mu}^{0,25} \chi^{0,5} y / \mu$,(6); $\chi$ is Karman constant; $E$ is empirical coefficient.

The task $E$ preserves hydrodynamically smooth walls. In the literature, there are a number of formulas for rough and completely rough walls, as well as an analysis of theoretical and field data [6]. Works I.E. Idelchik and A.G. Adamov [4,5] are interesting. There is a lot of work on this issue in foreign literature, many of these studies have been carried out experimentally and numerically $[10,11,12,13]$. Most of them have the following form:

$$
u^{+}=y^{+}, y^{+} \leq y_{m}^{+} \text {, }
$$

$$
\begin{aligned}
& u^{+}=A+\frac{1}{\chi} \ln \left[\frac{y^{+}-D^{+}}{B+C R^{+}}\right], y^{+}>y_{m}^{+} \\
& D^{+}=\rho C_{\mu}^{1 / 4} \chi^{1 / 2} \frac{D}{\mu} \\
& R^{+}=\rho C_{\mu}^{1 / 4} \chi^{1 / 2} \frac{y_{0}}{\mu} .
\end{aligned}
$$

where $A, B$ and $C$ are constants; $D$ is displacement thickness; $y_{0}=\Delta_{\ni}$ is equivalent roughness height, according to experiments $\Delta_{\ni}=0,03 \mathrm{MM}$.

The value $y_{m}^{+}$satisfies the equation:

$y_{m}^{+}-\frac{1}{\chi} \ln \left(E y_{m}^{+}\right)=0$.

The last two quantitative characteristics are illustrated in Figure 1. The value $D$ is such that the fluid velocity decreases to zero at a distance - $D+y_{0}$ from the wall. The value $y_{m}^{+}$depends on the formula used. For a completely rough wall $y_{m}^{+}=0$, since equation (3) is not used. The value of the constants depends on the type of 
Safar Z. Zainudinov et al., International Journal of Emerging Trends in Engineering Research, 8(5), May 2020,1605 - 1609

flow and the type of roughness. According to the research of A.D. Altshul [1] the possible discrepancies in the calculation using various formulas are insignificant, compared with the errors that usually occur due to the uncertainty in the choice of roughness values. The performed measurements by Nikuradze [9] of flows in pipelines having a roughness of sand gave the following constant values $A=0,85, B=0, C=1, D=0$.

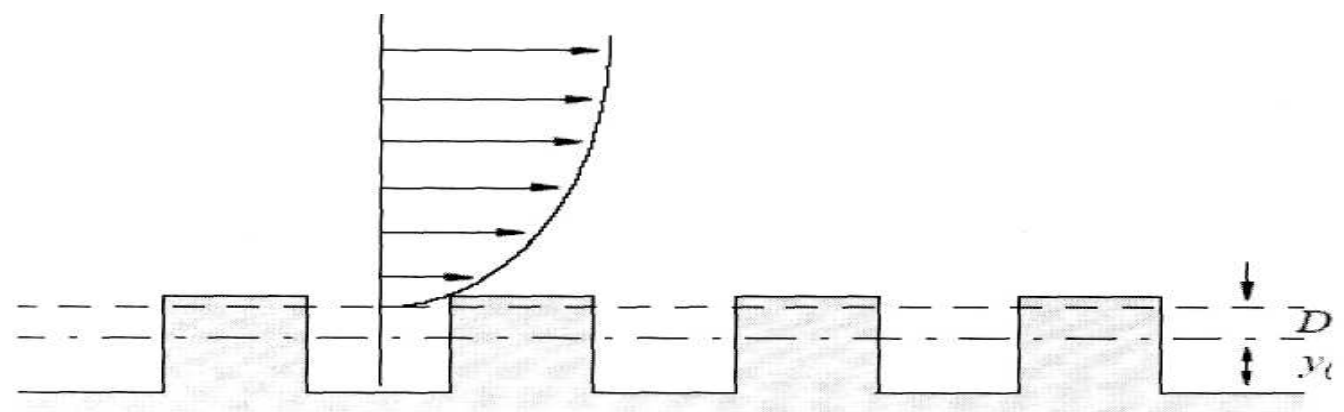

Figure 1: Illustration of a typical roughness element

The main condition for the verification (verification) of the model was its comparison with the actual experimental values. A comparative analysis was performed for each series of experiments by comparing the pressure fields of the mathematical model and the data that were obtained as a result of a hydraulic field experiment.

Verification of the experimental piezometric pressure values along the waterway route with the pressure values of the mathematical model of the flow for one of the options is shown in Figure 2, which shows a graph of the piezometric pressure changes in pascals along the length of the water pipe, where the experimental values are indicated by dots and piezometric pressures plotted by lines numerical model.

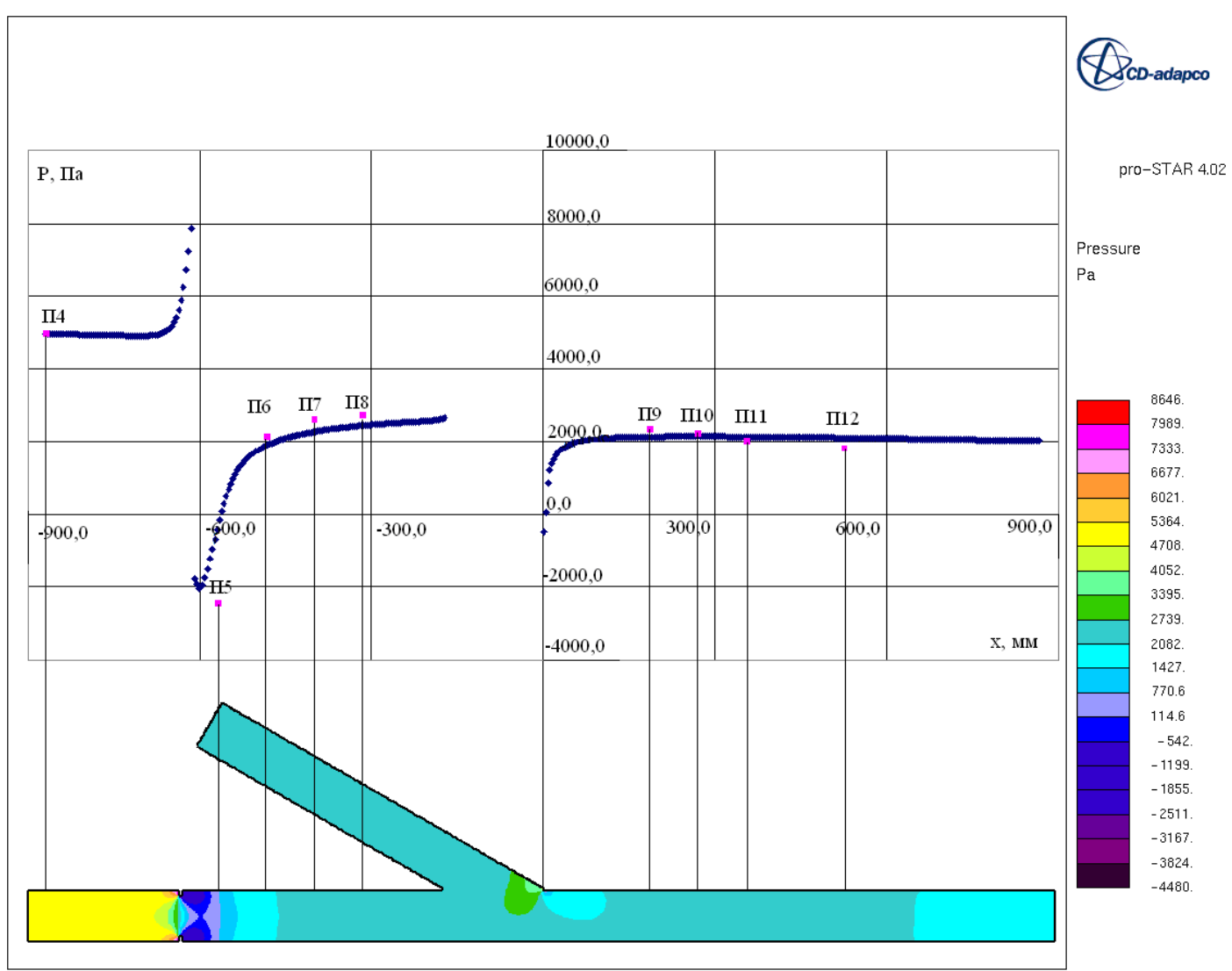

Figure 2 : Illustration of changes in piezometric pressure and pressures obtained using IT technology 


\section{CONCLUSION}

Verification of the data of local losses obtained as a result of a physical experiment and the data of mathematical modeling of the pressure flow in the CD-adapco STARCD application program shows their good coincidence when numerically solving the system of differential Navier-Stokes equations in a stationary turbulent flow.

A local decrease in piezometric pressure for all types of local resistances in the control range $a / d=0,1 \div 0,4$, with the formation of a vortex zone, is observed according to mathematical modeling in the considered local resistance unit, as well as according to the actual experiment. The piezometric pressure behind local resistances varied from 100 to $600 \mathrm{~mm}$ of a water column of liquid. The closure of the $a / d$ local resistance valves for the flow rate $Q=21 \mathrm{l} / \mathrm{s}$ and the Reynolds number $\operatorname{Re}=2 \cdot 10^{5}$ in the experimental and mathematical models corresponded to the nature of the flow when the pressure flow behind the local resistance is able to maintain the dynamic vacuum that forms immediately after the local resistance. In almost all cases, there was also a local increase and subsequent decrease in piezometric pressure in the interface between the tee branch and the transit branch of the water conduit.

Thus, the pressure fields for all considered cases obtained as a result of mathematical modeling showed good agreement with the data obtained experimentally. In all cases, the discrepancies between the experiment and the data of mathematical modeling were within $2-4 \%$.

\section{REFERENCES}

1. A.D. Altshul. Hydraulic resistance: textbook: 2nd edition. Moscow: Nedra, 1982. 224 p.

2. E.V. Andreev, V.Ya. Zharnitsky. Analysis of the interaction of the ice sheet with the hydraulic structure. Environmental Engineering. 2019. No. 4. Pp.5-10.

3. D.M. Benin, V.L. Snezhko. IT infrastructure of water enterprises: a training manual. Moscow: UMTS Triada LLC, 2020. 195 p.
4. I.E. Idelchik, G.A. Adamov. An experimental study of the resistance of plywood pipes of round and square sections in a fully developed turbulent flow. MAP works No. 670. Moscow, 1948. 27 p.

5. I.E. Idelchik, G.A. Adamov. Experimental study of turbulent flow in the initial sections of straight pipes of round and square sections: MAP Technical Reports No. 124. Moscow, 1948. 14 p.

6. V.V. Kazennov, A.V. Mishuev. Hydraulic resistance of pressure head pipelines of power plants. Scientific and technical journal Vestnik MGSU. - 2008. No. 1. Pp. 210 215. ISSN 1997-0935.

7. M.S. Paliivets, I.I. Abdullaev, V.L. Snezhko. The possibility of applying the injection effect in culverts of hydraulic structures. Environmental Engineering. 2019. No. 4. Pp. 55-60.

8. M.S. Paliivets. Mutual influence in reinforcing elements and nodes of pressure water conduits: monograph. Saarbrucken, Deutschland: LAP LAMBERT Academic Publishing, 2013. 216 p. Bibliography: p. 196 - 216. ISBN 978-3-659-43590-4.

9. J. Nikuradse. Strömungsgesetze in rauhen Rohren. Forschungsheft 361. Beilage zu "Forschung auf dem Gebiete des Ingenieurwesens", Ausgabe B, Bd. 4, Berlin. 1933. Pp.1- 22.

https://doi.org/10.1007/BF02716946

10. Shuisheng He, J.Gotts. Calculation of Friction Coefficients for Noncircular Channels. J. Fluids Eng. 2004. Volume 126. P. 986 - 992. doi:10.1115/1.1845479.

11. Y. Shimizu, Y. Futaki, C. S. Martin. Secondary Flow and Hydraulic Losses Within Sinuous Conduits of Rectangular Cross Section. J. Fluids Eng. 1992. Volume 114. Pp. 426-434. doi:10.1115/1.2910072.

12. A. Taylor, J. H. Whitelaw, M. Yianneskis. Curved Ducts With Strong Secondary Motion: Velocity Measurements of Developing Laminar and Turbulent Flow. J. Fluids Eng. 1982. Volume 104. Pp. 220 - 230. doi:10.1115/1.3241850.

13. J. Weisbach. Lehrbuch der technischen Mechanik. Berlin. 1875. 320 p. 